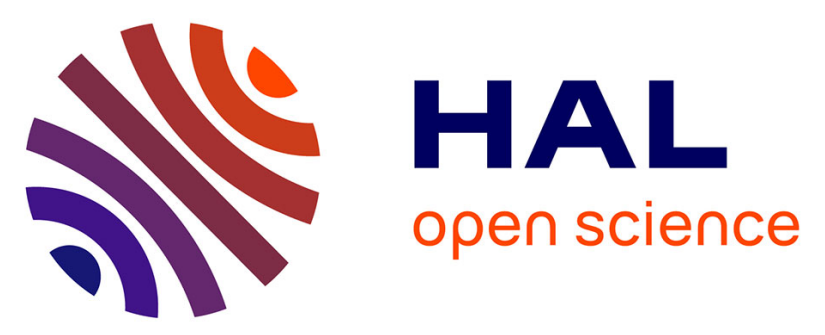

\title{
Local Texture Anisotropy as an Estimate of Muscle Quality in Ultrasound Imaging
}

Guillaume J.R. Dubois, Damien Bachasson, Lilian Lacourpaille, Olivier Benveniste, Jean-Yves Hogrel

\section{- To cite this version:}

Guillaume J.R. Dubois, Damien Bachasson, Lilian Lacourpaille, Olivier Benveniste, Jean-Yves Hogrel. Local Texture Anisotropy as an Estimate of Muscle Quality in Ultrasound Imaging. Ultrasound in Medicine \& Biology, 2018, 44 (5), pp.1133-1140. 10.1016/j.ultrasmedbio.2017.12.017 . hal-01962400

\section{HAL Id: hal-01962400 \\ https: / hal.sorbonne-universite.fr/hal-01962400}

Submitted on 14 Jan 2019

HAL is a multi-disciplinary open access archive for the deposit and dissemination of scientific research documents, whether they are published or not. The documents may come from teaching and research institutions in France or abroad, or from public or private research centers.
L'archive ouverte pluridisciplinaire HAL, est destinée au dépôt et à la diffusion de documents scientifiques de niveau recherche, publiés ou non, émanant des établissements d'enseignement et de recherche français ou étrangers, des laboratoires publics ou privés. 


\title{
LOCAL TEXTURE ANISOTROPY AS AN ESTIMATE OF MUSCLE QUALITY IN ULTRASOUND IMAGING
}

\author{
Guillaume J.R. Dubois, * Damien Bachasson, ${ }^{*}$ Lilian Lacourpaille, ${ }^{\dagger}$ \\ Olivier Benveniste, ${ }^{*}$ and Jean-Yves Hogrel* \\ * Institute of Myology, Paris, France; ${ }^{\dagger}$ Laboratory "Movement, Interactions, Performance" (EA 4334), Faculty of Sport Sciences, \\ University of Nantes, Nantes, France; and Inflammatory Muscle and Innovative Targeted Therapies. Department of Internal \\ Medicine and Clinical Immunology, University Pierre et Marie Curie, AP-HP, GH Pitié-Salpêtrière, Paris, France
}

\begin{abstract}
This study introduces local pattern texture anisotropy as a novel parameter to differentiate healthy and disordered muscle and to gauge the severity of muscle impairments based on B-mode ultrasound images. Preliminary human results are also presented. A local pattern texture anisotropy index (TAI) was computed in one region of interest in the short head of the biceps brachii. The effects of gain settings and box sizes required for TAI computation were investigated. Between-day reliability was studied in patients with sporadic inclusion body myositis $(n=26)$. The ability of the TAI to discriminate dystrophic from healthy muscle was evaluated in patients with Duchenne muscular dystrophy and healthy controls $(n=16)$. TAI values were compared with a grayscale index (GSI). TAI values were less influenced by gain settings than were GSI values. TAI had lower betweenday variability (typical error $=2.3 \%$ ) compared with GSI (typical error $=2.3 \%$ vs. $8.3 \%$, respectively). Patients with Duchenne muscular dystrophy had lower TAIs than controls $(0.76 \pm 0.06$ vs. $0.87 \pm 0.03$, respectively, $p<0.05)$. At $40 \%$ gain, TAI values correlated with percentage predicted elbow flexor strength in inclusion body myositis $(R=0.63, p<0.001)$. The TAI may be a promising addition to other texture-based approaches for quantitative muscle ultrasound imaging. (E-mail: d.bachasson@institut-myologie.org)
\end{abstract}

Key Words: Quantitative muscle ultrasound, Texture analysis, Muscle quality, Echogenicity, Local pattern texture anisotropy, Muscle dystrophy, Inclusion body myositis.

\section{INTRODUCTION}

Quantitative muscle ultrasound imaging has been reported to be an effective and cost-saving technique for the non-invasive assessment of skeletal muscle structure (Simon et al. 2016). Quantitative assessment of mean muscle echo intensity (EI) using gray-scale or backscatter analysis of a region of interest (ROI) has been extensively used as a proxy measure to estimate muscle quality (i.e., levels of intramuscular fibrosis and fatty infiltration) (Pillen et al. 2009). This parameter is highly dependent on the ultrasound device and settings (Pillen and Van Alfen 2015; Zaidman et al. 2008). In contrast to mean EI and other first-order descriptors (e.g., standard deviation, skewness, kurtosis and entropy), higher-order texture features

Address correspondence to: Damien Bachasson, Institut de Myologie, Groupe hospitalier Pitié-Salpêtrière, Batiment Babinski, 47-83 Boulevard de l'Hôpital, 75651 Cedex 13 Paris, France. E-mail: d.bachasson@ @institutmyologie.org (e.g., Haralick features, Galloway features, local binary pattern) have been identified as promising tools for diagnosis, characterization and follow-up of muscle alterations (Konig et al. 2015; Martinez-Paya et al. 2017; Molinari et al. 2015; Sogawa et al. 2017; Weng et al. 2017). These descriptors may have the advantage of being less affected by variables such as intensity.

Normal ultrasound images obtained along the longitudinal axis of the muscle reveal the perimysium as parallel echogenic lines on a background of the hypoechoic muscle fascicles, as illustrated in Figure 1A. This main orientation of the signal can be easily identified with the eye at multiple scales. This phenomenon may be referred to as local texture anisotropy. In the normal muscle, local texture anisotropy may thus be considered high. As degenerative muscle changes intensify (i.e., increase fat content and fibrosis), this preferential orientation of the signal may be disrupted; that is, the local texture anisotropy is reduced. A proposed method for quantitatively revealing and characterizing local texture anisotropy consists 


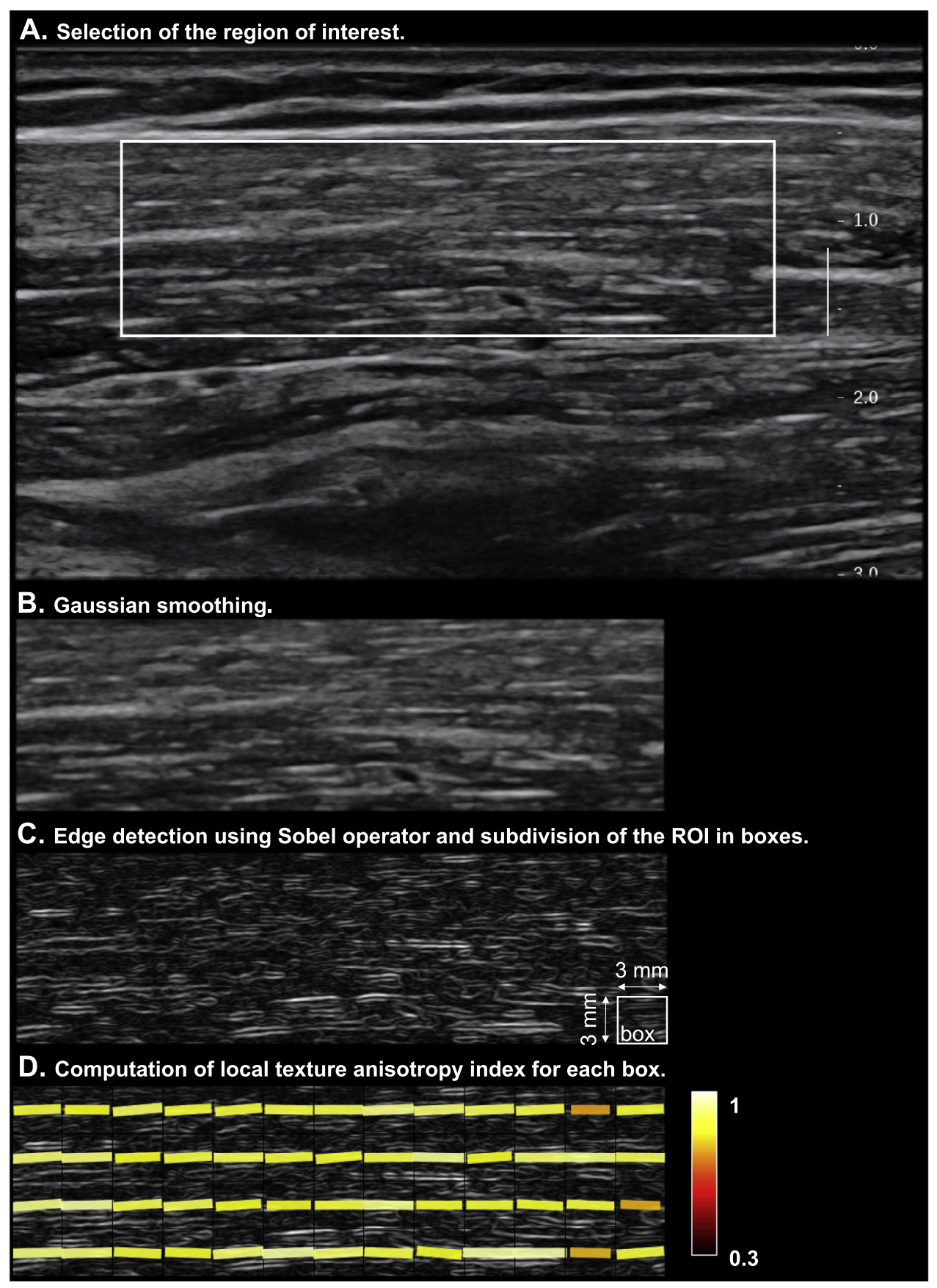

Fig. 1. Steps for computation of the texture anisotropy index (TAI). (A) Computation of the TAI is performed in a region of interest defined by the operator in the ultrasound image. (B) A Gaussian filter is applied. (C) An edge detection is performed using the Sobel operator in each $\mathrm{x}$ - and $\mathrm{y}$-direction, and a box size is defined (here, $3 \times 3 \mathrm{~mm}^{2}$ ). (D) The TAI for each box is computed. The vector size and color give the value of the TAI. The mean TAI is computed as the mean value of all TAI values for each box.

of dividing the image into analysis boxes; in each box, an ellipse (the inertia tensor) is fitted to the signal and determines the direction in which the signal is more present (Lehoucq et al. 2015). To the best of our knowledge, no previous report has investigated the potential of local texture anisotropy as an estimate of muscle quality.
The present article introduces a texture anisotropy index (TAI) as a texture-based approach to estimate muscle quality in B-mode ultrasound scans. This article is organized as follows: description of the methods, influence of settings for image acquisition and post-processing on the TAI (particularly with respect to the size of boxes defined 
for TAI computation), comparison with mean EI and presentation of preliminary human results.

\section{METHODS}

This study conformed to the Declaration of Helsinki and was approved by the local ethics committees. Experiments were performed between June 2013 and June 2016. All patients gave written informed consent.

\section{Muscle ultrasound imaging}

B-Mode ultrasound images were obtained in the short head of the biceps brachii using an Aixplorer Ultrasound device (V9.2, Supersonic Imagine, Aix-en-Provence, France) driving a 4-15 MHz linear transducer array (SL154, 256 elements, pitch $0.2 \mathrm{~mm}$ ). The biceps brachii, a fusiform muscle, was targeted because it is easy to access and because it is easy to standardize a robust imaging procedure. The belly of the short head of the biceps brachii was identified by one sonographer (G.J.R.D.) during transverse scanning at two-thirds of the distance between the acromion and the antecubital fossa. Then the probe was rotated and carefully aligned with the direction of muscle fascicles (Fig. 1A). Settings were defined as follows: musculoskeletal-muscle preset, penetration and HD mode; super compound: enabled; harmonic: disabled. Ultrasound images were acquired using five different settings with constant probe location: automatic time-gain compensation (Auto TGC), gains of $40 \%, 50 \%, 60 \%$ and $70 \%$ (with time-gain compensation maintained in the same [neutral] position for all depths). The depth setting was adapted for each participant during examination to display the entire muscle. Focus range was set on the entire depth for all patients. Images were stored as DICOM files and transferred to a computer for offline processing.

\section{TAI computation}

Analysis of images was performed in MATLAB (The MathWorks, Natick, MA, USA).

Area of measurement. The TAI was computed in one region of interest (ROI) selected in the muscle belly by the same experienced operator (G.J.R.D.) as illustrated in Figure 1A. The selected area was an $n \times m$ image (I).

Boxes. The size of the boxes was chosen in accordance with muscle fascicle thickness within the image plane and image resolution. To limit the effect of thick connective tissues within the muscle and considering a fascicle thickness of $\sim 2 \mathrm{~mm}$ evaluated on ultrasound images, different box sizes have been studied: $0.25,1,4,9,16$, 25 and $36 \mathrm{~mm}^{2}$.

Computational steps. A Gaussian smoothing (diameter: $5 \mathrm{px}, \sigma=1$ ) was applied (Fig. 1B) to reduce the effect of focal variations in signal intensity when estimating local texture anisotropy. Edge detection was performed using the Sobel operator (Sobel and Feldman 1968). Convolution operations $(\otimes)$ were performed along the $\mathrm{x}$-axis,

$$
G_{x}=\frac{1}{4} \cdot\left(\left[\begin{array}{lll}
-1 & 0 & 1 \\
-2 & 0 & 2 \\
-1 & 0 & 1
\end{array}\right] \otimes I\right)
$$

and the y-axis,

$$
G_{y}=\frac{1}{4} \cdot\left(\left[\begin{array}{ccc}
-1 & -2 & -1 \\
0 & 0 & 0 \\
1 & 2 & 1
\end{array}\right] \otimes I\right)
$$

An example of the obtained gradient magnitude $\left(G=\sqrt{G_{x}{ }^{2}+G_{y}{ }^{2}}\right)$ is provided in Figure 1C. TAI values were computed in equally spaced boxes. The size of each box was $u \times v$ (Fig. 1C, D). The local gradient matrix $W$ of a given box was defined by a matrix with $u \cdot v$ lines and two columns as, $W_{i 1}=G_{x_{i}}$ and $W_{i 2}=G_{y_{i}}$, with $i$ the pixel number in the box. The $W$ matrix was mean centered, and $W_{C}$ was computed as $W_{C_{i 1}}=W_{i 1}-\bar{W}_{1}$ and $W_{C_{i 2}}=W_{i 2}-\overline{W_{2}}$. The local covariance matrix $C$ was computed as the product between the transpose of $W_{C}$ and $W_{C}$ as $C={ }^{T} W_{C} \cdot W_{C}$. The two eigenvalues $\lambda$ of $C$ were retrieved and sorted with $\lambda_{1}>\lambda_{2}$. TAI for each box was defined as $\alpha=1-\lambda_{2} / \lambda_{1}$. Therefore, a TAI approaching 1 indicates the strong predominance of one orientation in the signal (i.e., strong local texture anisotropy) whereas TAI approaching 0 indicates a diffuse signal with no orientation predominant (i.e., weak local texture anisotropy) (Fig. 1D). The orientation of the fascicles relative to the probe has no effect on the TAI value. The mean TAI was defined as the mean of TAI values computed for each box. An illustration of TAI values in moderate (A) and severe (B) muscle impairments in two patients with inclusion body myositis (IBM) is provided in Figure 2.

\section{Quantification of mean muscle echo intensity}

On an $n \times m$ 8-bit gray-scale image, the intensity value of a pixel ranges from 0 to 255 . To correspond to the range of the TAI, a gray-scale index (GSI) was defined as the mean pixel intensity in a ROI and rescaled between 0 and 1 by dividing by 255 . To obtain the same slope between TAI and GSI values (lower GSI values should correspond to higher EI values), the GSI was computed as

$$
\mathrm{GSI}=1-\frac{\left(\frac{1}{n \cdot m} \sum_{i=1}^{n \cdot m} I_{i}\right)}{255}
$$

with $I_{i}$ the intensity of pixel $i$ in the selected area. 


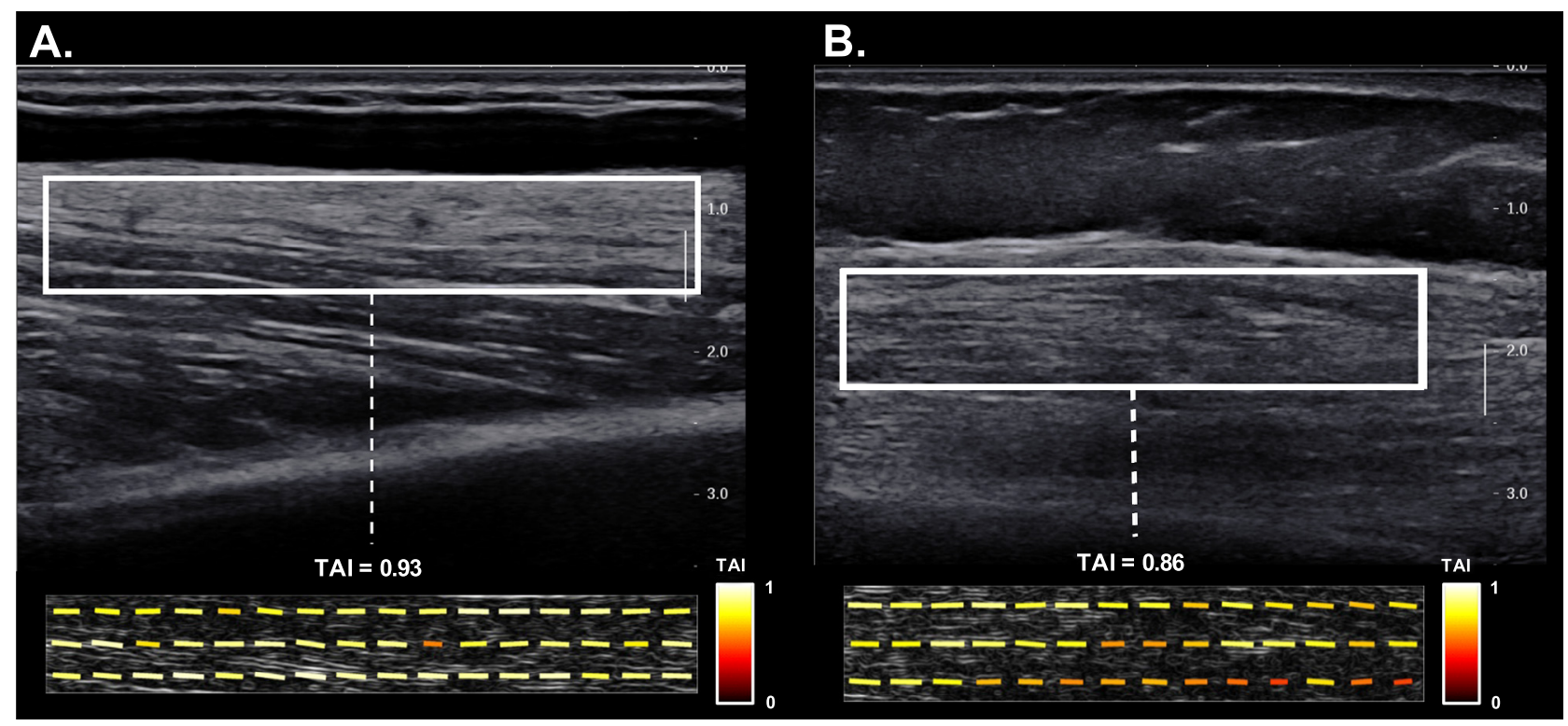

Fig. 2. Texture anisotropy index (TAI) values in moderate $(\mathrm{A})$ and severe $(\mathrm{B})$ muscle impairments in two patients with inclusion body myositis. Patients in (A) and (B) had maximal isometric elbow flexor strength corresponding to $69 \%$ and $42 \%$ of predicted values, respectively. Gray-scale index was 0.62 and 0.75 , respectively.

\section{Evaluation of the method}

The influence of gain setting and box size was evaluated in 26 patients with sporadic IBM (15 men and 11 women, age $=64.9 \pm 8.4 \mathrm{y}$, height $=1.67 \pm 0.08 \mathrm{~m}$, weight $=71.2 \pm 14.3 \mathrm{~kg}$ ). All patients had definite IBM (i.e., pathologic examination of their biopsies revealed fibers invaded by lymphocytes, vacuoles and amyloid deposits). The mean IBM weakness composite index (Benveniste et al. 2011) was $62 \pm 17$ (maximal score is 100). Ten patients were measured twice $7 \mathrm{~d}$ apart to assess the between-day reliability of both TAI and GSI values. To evaluate the ability of the TAI to estimate the magnitude of muscle impairments, the relationship between TAI values and muscle weakness level was investigated in patients with IBM. To this end, elbow flexion maximal voluntary isometric strength was assessed using an isokinetic dynamometer (Biodex, Biodex Medical, Shirley, NY, USA). Maximal peak torque was expressed as a percentage of predicted value using previously published equations (Hogrel et al. 2007). The ability of the TAI to discriminate dystrophic and healthy muscle was evaluated in 10 patients with genetically confirmed Duchenne muscular dystrophy (DMD, age $=12.1 \pm 6.1$ $\mathrm{y}$, height $=1.42 \pm 0.27 \mathrm{~m}$, weight $=41.9 \pm 23.7 \mathrm{~kg}$ ) and 10 matched healthy controls (age $=11.1 \pm 5.5 \mathrm{y}$, height $=1.47 \pm 0.22 \mathrm{~m}$, weight $=39.6 \pm 16.7 \mathrm{~kg}$ ) using the Auto TGC mode and $9-\mathrm{mm}^{2}$ boxes for TAI computation.

\section{Statistics}

Data within text and tables are presented as mean \pm standard deviation (SD) or mean $(95 \%$ confidence interval). The assumptions of normality and sphericity were confirmed using D'Agostino's $K^{2}$ test and Mauchly's test. Two-way analyses of variance (ANOVAs), box size $\times$ gain, were conducted to test main and interaction effects on TAI and GDI values with the Tukey HSD (honest significant difference) test. To assess the between-day reliability of TAI and GSI values, changes in the mean values of both sessions were computed, and paired $t$-tests were used to detect systematic bias (Atkinson and Nevill 1998). Typical error was expressed as a coefficient of variation $\left(\mathrm{CV}_{\mathrm{TE}}\right)$ to study absolute reliability. Given the ceiling effect associated with both TAI and GSI, intra-class correlation coefficients were not computed, and the Bland-Altman method with 95\% limits of agreements (LOA) was used to assess relative reliability. All analyses were performed with the Statistica Version 8.0 statistical software package (StatSoft, USA), and statistical significance was set at $p<0.05$.

\section{RESULTS}

Values of TAI and GSI for all box sizes and gain values tested are illustrated in Figure 3.

\section{Influence of gain settings}

TAI and GSI values for all gain settings are displayed in Figure 4. A significant effect of gain was found for TAI and GSI (both $p<0.001$ ). TAI values at $40 \%$ gain was significantly different from that obtained at $70 \%$ gain only $(-1.4 \pm 1.5 \%, p<0.05)$. TAI values at $60 \%$ and $70 \%$ gain were significantly smaller compared with TAI values 


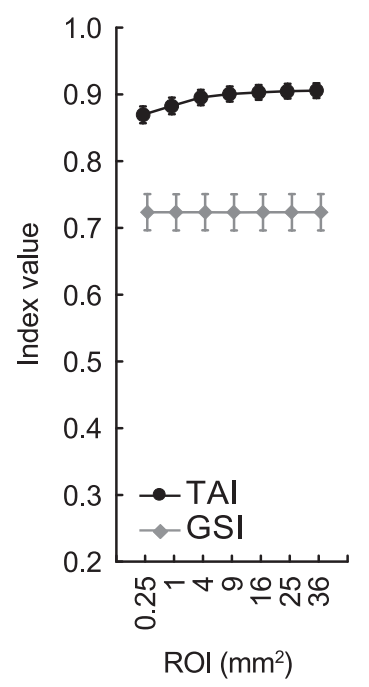

Auto TGC

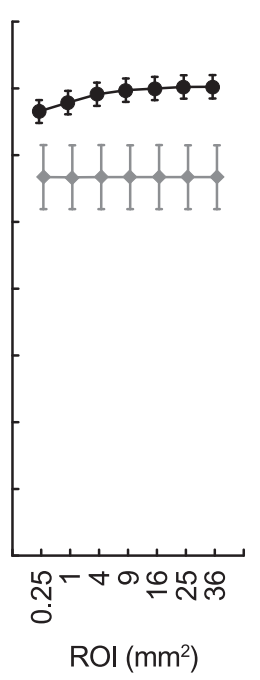

Gain $\mathbf{4 0 \%}$

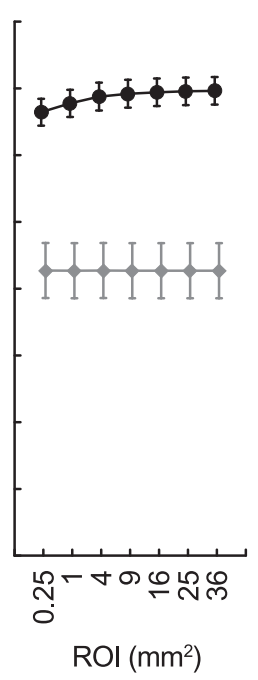

Gain $\mathbf{5 0 \%}$

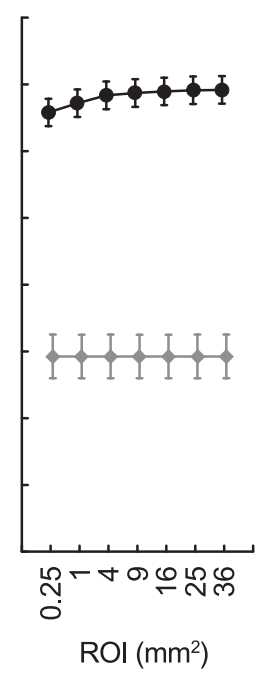

Gain $60 \%$

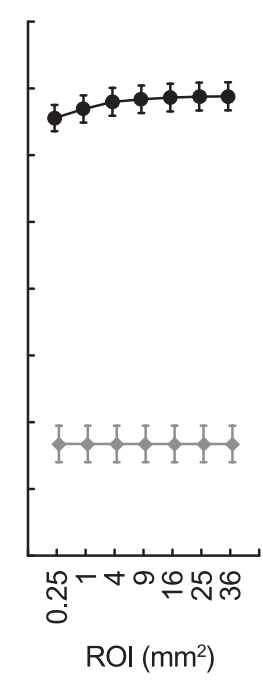

Gain $70 \%$

Fig. 3. Influence of gain setting for different box sizes on the texture anisotropy index (TAI) and gray-scale index (GSI). Auto $\mathrm{TGC}=$ automatic time-gain compensation; $\mathrm{ROI}=$ region of interest. Vertical bars denote 0.95 confidence intervals.

acquired using Auto TGC. GSI values at 50\%, 60\% and $70 \%$ gain were lower than that obtained at $40 \%$ gain or using Auto TGC ( $-52.2 \pm 2.4 \%$ at $70 \%$ compared with $40 \%$ gain).

\section{Influence of box size}

A significant effect of box size on TAI values was observed $(p<0.001)$. TAI values were significantly smaller with $0.25-, 1-$ and $4-\mathrm{mm}^{2}$ boxes compared to that obtained with $36-\mathrm{mm}^{2}$ boxes (Fig. 3). TAI values with 9-, $16-, 25-$ and $36-\mathrm{mm}^{2}$ boxes were similar (all $p$ values $>0.28)$. No significant effect of box size was found for the GSI $(p=0.39)$.

\section{Between-day reliability}

No significant change was found between test and retest values for both TAI and GSI values ( $p=0.92$ and $p=0.38$, respectively). Changes in mean, $\mathrm{CV}_{\mathrm{TE}}$ and LOA are provided in Table 1 and Bland-Altman plots in Figure 5.

\section{Preliminary human results}

Patients with DMD had significantly lower TAI and GSI values compared with controls (TAI: $0.76 \pm 0.06$ vs. $0.87 \pm 0.03$ and GSI: $0.65 \pm 0.09$ vs. $0.84 \pm 0.04$, respectively; both $p$ values $<0.05$ ) (Fig. 6). In patients with IBM, maximal voluntary elbow flexor strength was $26.4 \pm 12.7 \mathrm{Nm}$, corresponding to $50 \pm 19 \%$ of predicted
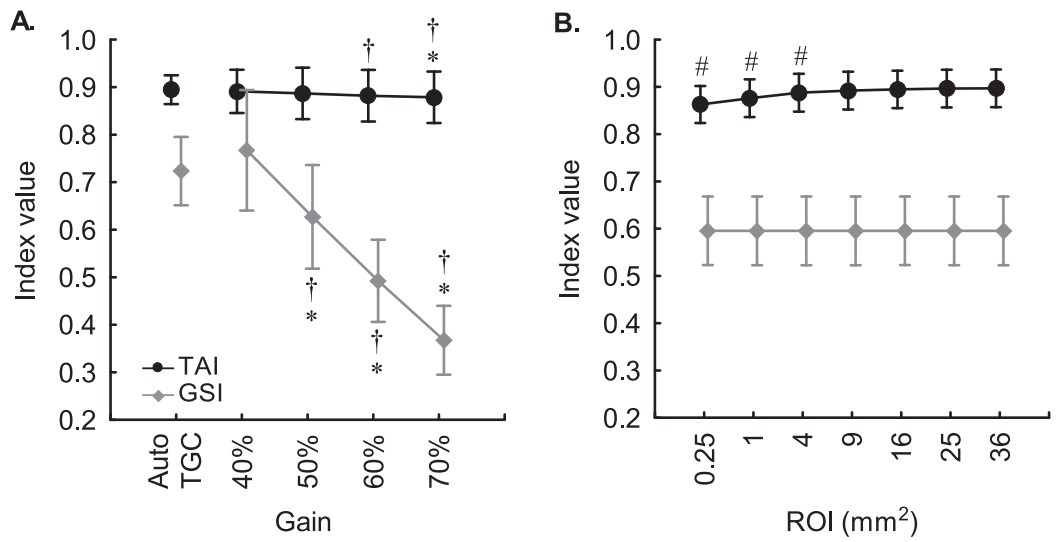

Fig. 4. Influence of gain setting (A) and box size (B) on texture anisotropy index (TAI) and gray-scale index (GSI). *Significantly different from $40 \%$ gain. "Significantly different from $36-\mathrm{mm}^{2}$ box. ${ }^{\dagger}$ Significantly different from Auto TGC. Vertical bars denote 0.95 confidence intervals. The TAI is less markedly affected by the gain setting compared with the GSI. A significant effect of box size was found for the TAI only. Auto TGC = automatic time-gain compensation. 
Table 1. Between-day reliability of the texture anisotropy index and gray-scale index

\begin{tabular}{lcccc}
\hline & & $\begin{array}{c}\text { Change in mean } \\
\text { (95\% confidence interval) }\end{array}$ & CV & Limits of agreement \\
\hline TAI & Mean \pm SD & $-0.001(-0.021$ to 0.020$)$ & $2.3(1.6$ to 4.2$)$ & -0.058 to 0.057 \\
GSI & $0.91 \pm 0.03$ & $-0.004(-0.065$ to 0.058$)$ & $7.9(5.4$ to 14.4$)$ & -0.172 to 0.164 \\
\hline
\end{tabular}

$\mathrm{TAI}=$ texture anisotropy index; GSI = gray-scale index; $\mathrm{CV}_{\mathrm{TE}}$, typical error expressed as a coefficient of variation; $95 \% \mathrm{CI}, 0.95 \mathrm{confidence} \mathrm{interval;}$ $\mathrm{SD}=$ standard deviation .

values. For the Auto TGC setting, TAI values correlated with percentage predicted strength $(R=0.63, p<0.001)$ (Fig. 7). At $40 \%$ gain, both TAI and GSI values correlated with percentage predicted strength $(R=0.63$, $p<0.001$, and $R=0.48, p<0.05$, respectively).

\section{DISCUSSION}

The present study introduced local texture anisotropy as a novel texture-based approach to estimate muscle quality on B-mode ultrasound images. The main findings are as follows: (i) the TAI can be easily and reliably computed from standard B-mode ultrasound images; (ii) the TAI was minimally influenced by the image gain settings; (iii) TAI values were significantly lower in patients with DMD than in healthy controls; (iv) TAI values and muscle weakness in patients with IBM were linearly related.

Our data indicated that TAI is less markedly influenced by image gain settings compared with GSI. The similarity between Auto TGC and 40\% and 50\% gain settings can be explained by the fact that when the Auto TGC setting was used, the gain was automatically set at $44.7 \pm 3.7 \%$ (Fig. 3). One potential explanation for reduced
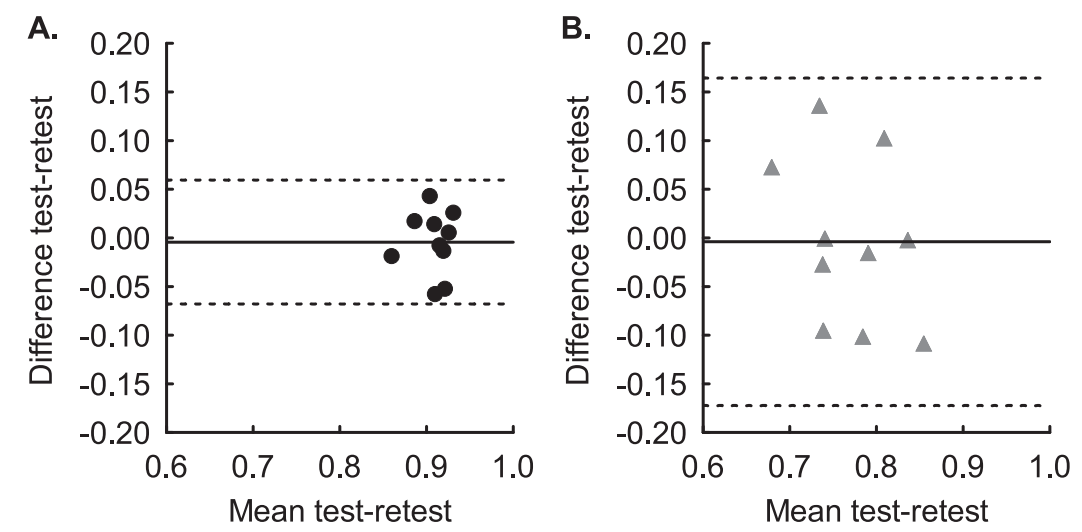

Fig. 5. Bland-Altman plots for between-day reliability of the texture anisotropy index (A) and gray-scale index (B) with $40 \%$ gain and a $9-\mathrm{mm}^{2}$ box. The plain line represents the bias, and the dashed lines, the limits of agreement.
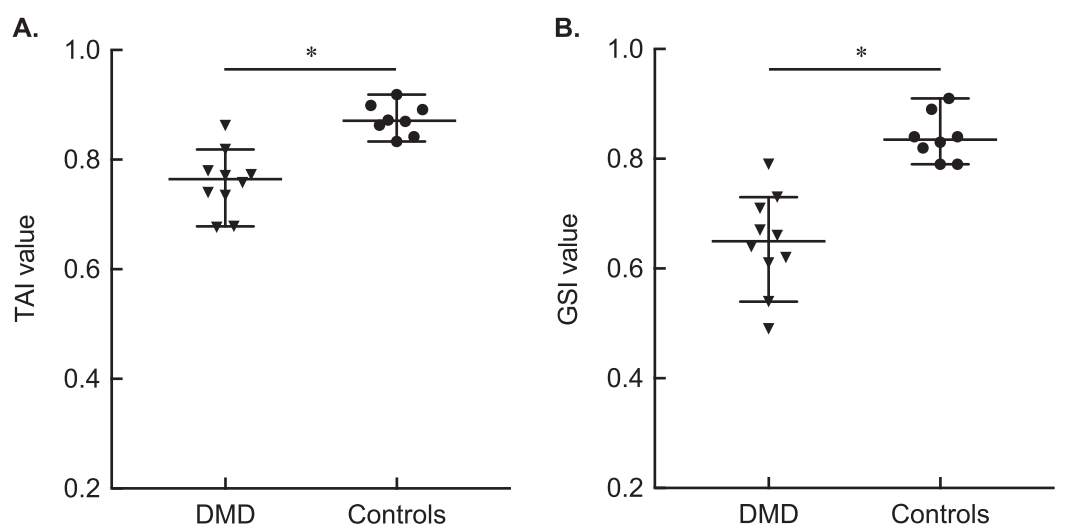

Fig. 6. Texture anisotropy index (TAI, A) and gray-scale index (GSI, B) in patients with Duchenne muscular dystrophy (DMD) and healthy controls (Controls). *Significant difference between DMD and controls. The TAI and the GSI were computed with a $9-\mathrm{mm}^{2}$ box. Vertical bars denote 0.95 confidence intervals. 

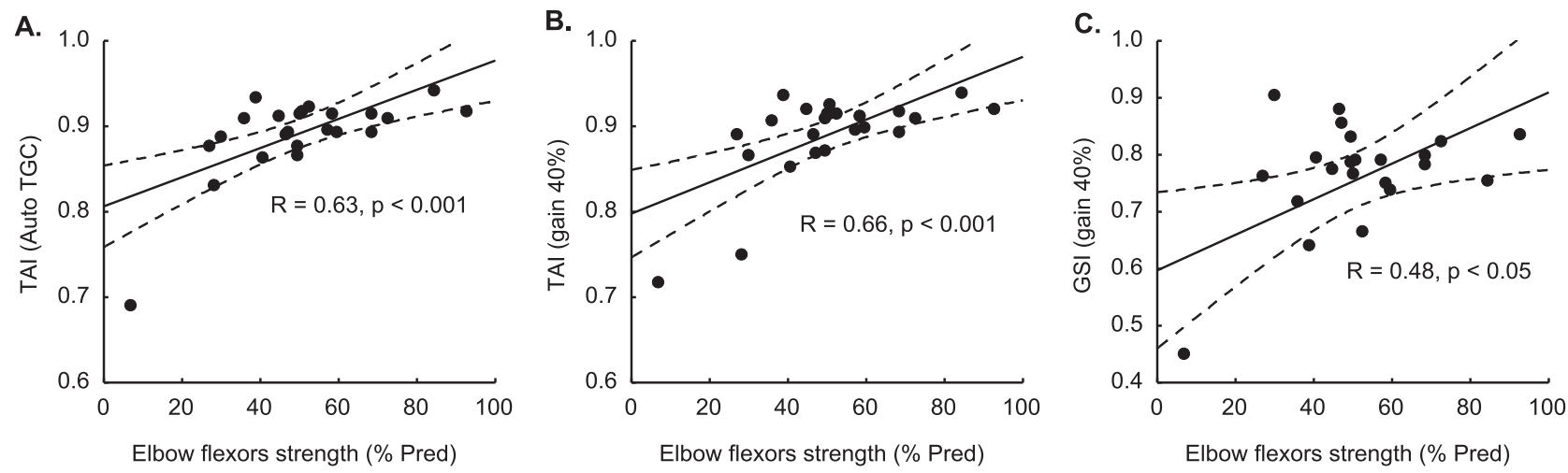

Fig. 7. Relation between the texture anisotropy index (TAI, A, B), gray-scale index (GSI, C) and maximal isometric elbow flexor strength in patients with inclusion body myositis. The TAI and GSI were computed using a 9-mm ${ }^{2}$ box. Pearson's correlation coefficient $(\mathrm{R})$ and significance are displayed. Auto TGC $=$ automatic time-gain compensation; \% Pred = percentage predicted values of maximal isometric elbow flexor strength.

TAI is that at the highest gain setting tested, anisotropic connective tissue bands are already at maximal pixel intensity, so they cannot increase, whereas the noise and diffuse isotropic backscatter speckle can continue to increase, thus lowering the effective TAI. The TAI was also affected by the size of the box used for computation; that is, smaller boxes were associated with lower TAI values (Fig. 4B). This may be explained by the fact that for ROI size $<9 \mathrm{~mm}^{2}$, one muscle fascicle and its corresponding perimysium could not be included in the ROI frame. In this case, the ROI was uniform (i.e., filled with either black or white) and the TAI value decreased toward 0 as it would do for an isotropic tissue (Fig. 2B). Consequently, the ROI must be larger than the fascicle diameter to avoid this effect. Analogously, box size was maintained lower than $36 \mathrm{~mm}^{2}$ to limit the effect of thick fascia or connective tissue crossing the image, which may significantly change the mean TAI. The use of Sobel edge detection and averaging the values over several boxes subsequently reduced these effects. This approach may also allow investigation of heterogeneous muscle changes by studying the distribution of TAI values within a target muscle.

Our data exhibited excellent between-day reliability of the TAI ( $\mathrm{CV}_{\mathrm{TE}} \sim 3 \%$ compared with $\sim 9 \%$ for the GSI) (Table 1), emphasizing the low dependency of the TAI on technological and operational confounding factors when using a standardized imaging protocol. The TAI should be applicable to most muscles commonly investigated in ultrasound imaging. However, and as previously performed with mean EI, the muscle dependence of TAI values and other potential influencing factors (e.g., age, sex, fitness level, type of muscle diseases) remain to be specifically investigated along with other first- and second-order descriptors (Konig et al. 2015; Martinez-Paya et al. 2017; Molinari et al. 2015; Sogawa et al. 2017).
As illustrated in patients with DMD using mean EI, the TAI also allows discrimination between dystrophic and healthy muscles (Fig. 5), in line with previous reports using mean EI and other texture-based analyses (Jansen et al. 2012; Zaidman et al. 2010). Extensive robustness analysis in more muscles with larger numbers of patients and controls remains to be conducted to evaluate the diagnostic validity of the TAI (Martinez-Paya et al. 2017; Sogawa et al. 2017). In patients with IBM, the correlation observed between muscle weakness and TAI values supports an association between local texture anisotropy and the magnitude of muscle changes, as previously observed using EI (Jansen et al. 2012; Nodera et al. 2016) and other texturebased approaches (Weng et al. 2017). Similarly to other metrics, such as mean EI, the TAI provides a non-specific estimate of muscle quality (Pillen and Van Alfen 2015), and data regarding tissue composition (e.g., fat infiltration, fibrosis) are thus required to identify the main factors influencing TAI values.

Together, these findings suggest that the TAI may be promising as an additional texture-based approach to diagnosis, characterization and follow-up of muscle alterations using ultrasound imaging. This approach may be combined with common first-order descriptor and complex higher-order texture features recently proposed in the field (Molinari et al. 2015; Sogawa et al. 2017).

Acknowledgments-We gratefully thank all the patients who participated in this study. This study was supported by the Association Française Contre Les Myopathies (AFM).

\section{REFERENCES}

Atkinson G, Nevill AM. Statistical methods for assessing measurement error (reliability) in variables relevant to sports medicine. Sports Med 1998;26:217-238. 
Benveniste O, Guiguet M, Freebody J, Dubourg O, Squier W, Maisonobe T, Stojkovic T, Leite MI, Allenbach Y, Herson S, Brady S, Eymard B, Hilton-Jones D. Long-term observational study of sporadic inclusion body myositis. Brain 2011;134:3176-3184.

Hogrel JY, Payan CA, Ollivier G, Tanant V, Attarian S, Couillandre A, Dupeyron A, Lacomblez L, Doppler V, Meininger V, Tranchant C, Pouget J, Desnuelle C. Development of a French isometric strength normative database for adults using quantitative muscle testing. Arch Phys Med Rehabil 2007;88:1289-1297.

Jansen M, van Alfen N, Nijhuis van der Sanden MW, van Dijk JP, Pillen S, de Groot IJ. Quantitative muscle ultrasound is a promising longitudinal follow-up tool in Duchenne muscular dystrophy. Neuromuscul Disord 2012;22:306-317.

Konig T, Steffen J, Rak M, Neumann G, von Rohden L, Tonnies KD. Ultrasound texture-based CAD system for detecting neuromuscular diseases. Int J Comput Assist Radiol Surg 2015;10:1493-1503.

Lehoucq R, Weiss J, Dubrulle B, Amon A, Le Bouil A, Crassous J, Amitrano D, Graner F. Analysis of image vs. position, scale and direction reveals pattern texture anisotropy. Front Phys 2015;2:84.

Martinez-Paya JJ, Rios-Diaz J, Del Bano-Aledo ME, Tembl-Ferrairo JI, Vazquez-Costa JF, Medina-Mirapeix F. Quantitative muscle ultrasonography using textural analysis in amyotrophic lateral sclerosis. Ultrason Imaging 2017;39:357-368.

Molinari F, Caresio C, Acharya UR, Mookiah MR, Minetto MA. Advances in quantitative muscle ultrasonography using texture analysis of ultrasound images. Ultrasound Med Biol 2015;41:2520-2532.

Nodera H, Takamatsu N, Matsui N, Mori A, Terasawa Y, Shimatani Y, Osaki Y, Maruyama K, Izumi Y, Kaji R. Intramuscular dissociation of echogenicity in the triceps surae characterizes sporadic inclusion body myositis. Eur J Neurol 2016;23:588-596.

Pillen S, Van Alfen N. Muscle ultrasound from diagnostic tool to outcome measure: Quantification is the challenge. Muscle Nerve 2015;52: 319-320.

Pillen S, van Dijk JP, Weijers G, Raijmann W, de Korte CL, Zwarts MJ. Quantitative gray-scale analysis in skeletal muscle ultrasound: A comparison study of two ultrasound devices. Muscle Nerve 2009;39: 781-786.

Simon NG, Noto YI, Zaidman CM. Skeletal muscle imaging in neuromuscular disease. J Clin Neurosci 2016;33:1-10.

Sobel I, Feldman G. A $3 \times 3$ isotropic gradient operator for image processing. Presented at a talk at the Stanford Artificial Intelligence Laboratory, Stanford University, Stanford, CA; 1968.

Sogawa K, Nodera H, Takamatsu N, Mori A, Yamazaki H, Shimatani Y, Izumi Y, Kaji R. Neurogenic and myogenic diseases: Quantitative texture analysis of muscle US data for differentiation. Radiology 2017;283:492-498.

Weng WC, Tsui PH, Lin CW, Lu CH, Lin CY, Shieh JY, Lu FL, Ee TW, Wu KW, Lee WT. Evaluation of muscular changes by ultrasound Nakagami imaging in Duchenne muscular dystrophy. Sci Rep 2017;7:4429

Zaidman CM, Holland MR, Anderson CC, Pestronk A. Calibrated quantitative ultrasound imaging of skeletal muscle using backscatter analysis. Muscle Nerve 2008:38:893-898.

Zaidman CM, Connolly AM, Malkus EC, Florence JM, Pestronk A. Quantitative ultrasound using backscatter analysis in Duchenne and Becker muscular dystrophy. Neuromuscul Disord 2010;20:805-809. 